\title{
The Effects of Resistant Starch Consumption in Adult Patients with Chronic Kidney Disease: A Systematic Review Protocol
}

\section{Kulwant Kingra}

University of Manitoba College of Medicine

\section{Rebecca Mollard}

University of Manitoba

\section{Sarah Curtis}

Seven Oaks General Hospital

\section{Maryam Shamloo}

University of Manitoba

\section{Nicole Askin}

University of Manitoba

Navdeep Tangri ( $\nabla$ ntangri@sogh.mb.ca )

University of Manitoba College of Medicine

\section{Dylan MacKay}

University of Manitoba

\section{Protocol}

Keywords: chronic kidney disease, resistant starch, uremic toxins, oxidative stress, inflammation

Posted Date: October 19th, 2020

DOI: https://doi.org/10.21203/rs.3.rs-93107/v1

License: (c) (i) This work is licensed under a Creative Commons Attribution 4.0 International License. Read Full License 


\section{Abstract}

Background: Dietary modifications represent an important intervention to reduce the risk of chronic disease. Appropriate dietary changes can be simple to implement and are usually suggested as first line recommendations for many types of chronic disease management including diseases like hypertension and diabetes. Resistant starch is a non-digestible carbohydrate which passes through untouched to the colon where it becomes a digestible substrate for beneficial colonic bacteria. The normal gut flora becomes damaged in patients with chronic kidney disease with an additional buildup of uremic toxins and resulting intra-renal inflammation. Resistant starch supplementation has been studied for its effects on reducing harmful metabolite buildup through a restoration of normal gut flora. The following systematic review aims to compile the evidence of resistant starch use in adult patients with chronic kidney disease to answer whether or not the dietary intervention can reduce the progression of renal disease.

Methods: We will perform a literature search with a knowledge synthesis librarian including the following databases: MEDLINE, Cochrane Central, Embase, CINAHL, and Web of Science. Collected data will be extracted and organized in MS Excel 2019. The extraction of the data will include study details, study population details, intervention details, and outcome details.

Discussion: Results from this systematic review may help to determine a simple and effective method for prolonging the need for renal replacement therapy through resistant starch supplementation. Lack of evidence may highlight the need for additional trials or new interventions.

PROSPERO ID: Currently being assessed, ID: 203138.

\section{Background}

Dietary changes are considered first line non pharmacological options for chronic disease prevention and management in patients with diabetes and hypertension. Concerns have been growing in regards to foods high in digestible starch as they may contribute to an increasing risk of chronic diseases (1). These types of diets may result in dysbiosis or changes in the gut microbiota and this may play a large role in progressively worsening kidney disease (1).

Chronic kidney disease (CKD) can be defined as a reduction in kidney function as shown via a glomerular filtration rate (GFR) less than $60 \mathrm{~mL} / \mathrm{min}$ per $1.73 \mathrm{~m}^{2}$, or identification of any markers related to kidney damage, or both, of a minimum of 3 months, regardless of the precipitating cause (2). Hemodialysis (HD) is a treatment reserved for patients with kidney failure (KF), or in select cases of acute kidney injury, providing lifesaving renal replacement therapy. Prior studies in patients on HD have shown reduced amounts of beneficial bacteria and higher fecal concentrations of uremic toxins (3).

Dysbiosis is a source of inflammation which can increase the likelihood of renal disease progression (4). Chronic low-grade intrarenal inflammation can negatively affect microcirculation and blood perfusion 
ultimately inducing kidney damage (4). The inflammatory response is also related to oxidative stress and the production of reactive oxygen species (ROS) which can lead to further renal tubular injury (4). Dietary strategies have been designed to help combat the concerns with inflammation and oxidative stress that occur due to changes in gut microbiota. Resistant starch (RS) is a carbohydrate that cannot be broken down in the small intestine by digestive a-amylases, as opposed to soluble fibre for example (1)(3). RS instead passes to the colon whereby it begins to ferment and act as a digestible substrate for beneficial colonic bacteria (1)(3). RS can be divided into 4 different types and are characterized as either type I, type 2 , type 3 , or type 4 (5). Types 1-4 are differentiated based on their physical properties as well as which food sources they originate from (5). Important effects of RS through gut bacteria modulation include a reduction of harmful metabolite build up and the production of short chain fatty acids (SCFA), which are vital for their positive effects on immunity and inflammation (1)(3).

RS may also have an important role in reducing the amounts of toxic nitrogenous waste buildup which are known to accumulate in patients with CKD (3). Some of the main culprit toxins include urea, p-cresol sulfate, and indoxyl sulfate (3). Both p-cresol sulfate and indoxyl sulfate have been associated with an increased risk of mortality and cardiovascular events in patients with CKD as well as tubular and kidney damage, which would be linked with further progression of renal disease (6).

Due to the ease of administration and relatively low cost of RS it can become an important part of the treatment regimen of patients with CKD. Thus, the main objective of this systematic review is to assess the efficacy and tolerability of RS versus placebo in reducing the progression of renal disease in adult patients with CKD. This information will hopefully provide a basis and foundation for further clinical studies for resistant starch use in patients with CKD.

\section{Research Question}

Does the consumption of resistant starch vs. placebo reduce the progression of renal disease in adult patients with chronic kidney disease?

\section{Methods}

\section{Study Selection}

A knowledge synthesis librarian will develop a literature search strategy for MEDLINE. Subsequently, this will be reviewed by a second and independent librarian using the PRESS checklist. The search strategy will then be modified and adjusted for use in other databases (Cochrane Central, Embase, Web of Science, CINAHL) and this will be completed by a knowledge synthesis librarian. The appropriate citations will be screened for eligibility on Rayyan (Rayyan, Doha, Qatar) (7) by two independent reviewers. Ineligible citations will be recorded and the number and reason for exclusion will be documented at the full-text article screening stage. Disagreements on the eligibility of citations will be discussed by two systematic reviewers with a third reviewer to adjudicate, if required. 


\section{Eligibility criteria}

The following studies will be included:

1. Population: adults ( $\geq 18$ years of age) with chronic kidney disease in outpatient setting

2. Intervention: resistant starch supplementation

3. Comparator: placebo

4. Outcomes:

Primary: reduced decline in glomerular filtration rate from baseline through the end of the trial

Secondary: reduced uremic toxin buildup ( $p$-cresol, indoxyl sulfate) from baseline through the end of the clinical trial; reduced inflammatory markers in kidney disease (TNFa, CRP, IL-6) from baseline through the end of the clinical trial; improvement in self-reported symptom scores

Safety: determine tolerability of resistant starch (bloating, diarrhea, etc.)

5. Study design: randomized control trials that compare RS to placebo, no treatment, or the standard of care

6. No limitations on publication year

7. Full-text manuscript in English language (for feasibility)

\section{Data extraction}

Data extraction will be done through the use of forms developed in MS Excel 2019 (Microsoft Corporation, Redmond, WA, USA) (8). Extracted data will also be stored and managed in MS Excel. The data from all included studies will be extracted independently by two systematic reviewers. Any concerns or disagreements will be discussed and settled between the two reviewers. A third reviewer will adjudicate if the issues are still not resolved appropriately. The following data will be extracted from the included studies:

Study details: name of first author, year study was conducted, year of publication, country, setting, population demography, study size, and funding source

Study population details: type of population (for example, adults), age, sex distribution, health and socioeconomic status

Intervention details: name, type, method of intervention, measure (amount/extent), duration

Outcome details: Data will be extracted at the end of the trial and at the longest reported follow-up.

\section{Assessment of risk of bias}

The risk of bias will be checked through the Cochrane Risk of Bias Tool 2.0 (9). The tool will provide an assessment score in the forms of high, some concerns, and low risk of bias in each of the following 
areas: bias arising from the randomization process, bias due to deviations from intended interventions, bias due to missing outcome data, bias in measurement of the outcome, and bias in selection of the reported result. Any disagreements or issues will be discussed by the two reviewers or by involving a third reviewer if required.

\section{Data analysis}

Our plan will be to conduct meta-analysis wherever possible, using a random effects model executed in RevMan (Version 5.4) (10). Continuous data will be presented as standardized mean differences with $95 \%$ confidence intervals. Dichotomous data will be assessed and shown as a risk ratio. Statistical heterogeneity will be assessed between the included studies by using the l-squared statistic $\left(\mathrm{I}^{2}\right)$. Our assessment of publication bias will be completed through the use of funnel plots of effect size versus sample size for each included study and using Egger's regression test.

\section{Study Outcome Dissemination}

We will aim to circulate our results in the form of a peer-reviewed academic publication. We will also present our results at the appropriate academic meetings and conferences.

\section{Discussion}

A review of the literature is necessary to help determine if there is sufficient evidence to support the use of RS supplementation in patients with CKD. Patients with kidney disease often suffer a reduced quality of life secondary to symptoms related to worsening kidney function. RS may become a cheap and novel strategy to reduce the progression of kidney disease and could be easily incorporated as a part of treatment. It will be important to assess the different efficacy and safety outcomes of RS in order to evaluate the potential effectiveness of its use. The information attained from this systematic review will hopefully lead to further advancements in the management of late stage CKD. A lack of evidence will potentially propagate the need for further clinical trials.

\section{Abbreviations}

$\mathrm{ESRD}=$ end stage renal disease

CKD = chronic kidney disease

GFR = glomerular filtration rate

eGFR = estimated glomerular filtration rate

ROS $=$ reactive oxygen species

SCFA = short chain fatty acid 
RS = resistant starch

$\mathrm{CDIC}=$ chronic disease innovation centre

SOGH = Seven Oaks General Hospital

\section{Declarations}

Ethics approval and consent to participate:

Not applicable

\section{Consent for publication:}

Not applicable

\section{Availability of data and materials:}

No datasets have been generated related to this

\section{Competing interests:}

None to report

\section{Funding:}

University of Manitoba and Seven Oaks Chronic Disease Innovation Centre

\section{Authors' contributions:}

Each author has contributed greatly towards the generation of this paper. All authors have reviewed and approved the final manuscript.

\section{Acknowledgments:}

Not applicable

\section{References}

1. Birt DF, Boylston T, Hendrich S, Jane J-L, Hollis J, Li L, et al. Resistant Starch: Promise for Improving Human Health. Adv Nutr. 2013;4(6):587-601.

2. Webster AC, Nagler E V., Morton RL, Masson P. Chronic Kidney Disease. Lancet [Internet]. 2017;389(10075):1238-52. Available from: http://dx.doi.org/10.1016/S0140-6736(16)32064-5

3. Snelson M, Kellow NJ, Coughlan MT. Modulation of the Gut Microbiota by Resistant Starch as a Treatment of Chronic Kidney Diseases: Evidence of Efficacy and Mechanistic Insights. Adv Nutr. 
2019;10(2):303-20.

4. Mihai S, Codrici E, Popescu ID, Enciu AM, Albulescu L, Necula LG, et al. Inflammation-related mechanisms in chronic kidney disease prediction, progression, and outcome. J Immunol Res. 2018;2018.

5. Sajilata MG, Singhal RS, Kulkarni PR. Resistant Starch - A Review. Compr Rev Food Sci Saf. 2006;5(1).

6. Vanholder R, Schepers E, Pletinck A, Nagler E V., Glorieux G. The uremic toxicity of indoxyl sulfate and p-cresyl sulfate: A systematic review. J Am Soc Nephrol. 2014;25(9):1897-907.

7. Rayyan QCRI, the Systematic Reviews web app [Internet]. [cited 2020 July 19]. Available from: https://rayyan.qcri.org/welcome

8. Use of Microsoft Copyrighted Content I Intellectual Property [Internet]. [cited 2020 July 19]. Available from: https://www.microsoft.com/en-us/legal/intellectualproperty/permissions/default.aspx

9. Sterne JAC, Savović J, Page MJ, Elbers RG, Blencowe NS, Boutron I, et al. RoB 2: a revised tool for assessing risk of bias in randomised trials. BMJ [Internet]. 2019 Aug 28 [cited 2020 Jan 13];366. Available from: https://www.bmj.com/content/366/bmj.14898

10. RevMan 5 [Internet]. [cited 2020 July 19]. Available from: https://community.cochrane.org/help/tools-and-software/revman-5

\section{Search Strategy}

\section{MEDLINE Preliminary Search Strategy}

1. exp renal dialysis/ or renal replacement therapy/ or intermittent renal replacement therapy/ or hybrid renal replacement therapy/ or hemofiltration/ (121272)

2. exp renal insufficiency, chronic/ or renal insufficiency/ or uremia/ (144063)

3. (((renal or kidney) adj3 (support or therap* or replace*)) or dialys* or dialyz* or h?emodial* or $\mathrm{h}$ ? emodiafiltrat* or h?emofiltrat* or capd or ccpd or apd or rrt* or irrt* or pirrt* or ihd* or hirrt* or hybrid extracorporeal or hybrid extra-corporeal or sled or edd or sledd or sleddf or hdf or olhdf or avvh or avvhdf).tw,kf. (201561)

4. (((chronic or endstage or end-stage or advanced) adj2 (kidney or renal)) or ckd or ckf or crf or crd or esrf or eskf or esrd or eskd or ur?emi*).tw,kf. (166100)

5. or/1-4 (361858)

6. exp starch/ or exp dietary fiber/ (61197)

7. (starch* or "high amylose" or retrograd* amylose or rs 1 or rs 2 or rs 3 or rs 4 or rs 5 or hamrs* or hamsr* or ham-rs* or hamsr* or ((nondigestible or non-digestible or indigestible or non-soluble or nonsoluble or insoluble) adj2 carbohydrate*) or ndc* or dietary fiber*).tw,kf. (50325)

8. exp fabaceae/ or plantago/ or musa/ or whole grains/ or exp poaceae/ (195135) 
9. (plantain* or green banana* or bean or beans or pea or peas or legume* or lentil* or chickpea* or whole grain* or wholegrain* or wholemeal* or oats or barley* or rice or potato starch or hi-maize or durum or semolina).tw,kf. (129408)

10. or/6-9 (335989)

11. randomized controlled trial.pt. (508716)

12. controlled clinical trial.pt. (93742)

13. Clinical Trials as topic/ (191833)

14. ((singl* or doubl* or trebl* or tripl*) adj (blind* or dumm* or mask*)).tw,kf. (172983)

15. PLACEBOS/ (34944)

16. (placebo* or sham).tw,kf. (299332)

17. (randomized or randomised or randomly or rct).tw,kf. (894606)

18. trial.ti. (220746)

19. or/11-18 (1422899)

20. 5 and 10 and 19 (278)

21. exp animals/ not humans/ (4712675)

22. 20 not 21 (229)

23. (adolescent/ or exp child/ or exp infant/) not exp adult/ (1873919)

24. 22 not $23(225)$

\section{Supplementary Files}

This is a list of supplementary files associated with this preprint. Click to download.

- AdditionalFile1PRISMAP.docx 